\title{
Bayesian network structure and predictability of autistic traits
}

\author{
Giovanni Briganti ${ }^{1}$, Donald R. Williams ${ }^{2}$, Joris Mulder ${ }^{3}$, and Paul Linkowski ${ }^{1}$ \\ ${ }^{1}$ Université libre de Bruxelles \\ ${ }^{2}$ University of California, Davis \\ ${ }^{3}$ Tilburg University
}

November 7, 2020

\begin{abstract}
The aim of this work is to explore the construct of autistic traits through the lens of network analysis with recently introduced Bayesian methods. A conditional dependence network structure was estimated from a data set composed of 649 university students that completed an autistic traits questionnaire. The connectedness of the network is also explored, as well as sex differences among female and male subjects in regard to network connectivity. The strongest connections in the network are found between items that measure similar autistic traits. Traits related to social skills are the most interconnected items in the network. Sex differences are found between female and male subjects. The Bayesian network analysis offers new insight on the connectivity of autistic traits as well as confirms several findings in the autism literature.
\end{abstract}

Keywords: autism; network analysis; students

This paper was peer-reviewed and accepted for publication in Psychological Reports Corresponding author: Giovanni Briganti, giovanni.briganti@hotmail.com

\section{Introduction}

For the past two decades, there has been a growing interest in psychiatric research for the classification and measurement of autistic traits (Volkmar et al., 2009). The Diagnostic and Statistical Manual of Mental Disorders (DSM-V) has defined a framework for autistic traits: the Autism Spectrum Disorder (ASD), that includes child autism, Kanner's infantile autism, high-functioning autism, and Asperger Syndrome.

ASD is understood as a neurological disorder as well, though potential etiopathogenic mechanisms at different levels of scientific explanation are limited: that ASD retains a moderate rate of heritability (37\%-90\%) is largely accepted, but the underpinnings of core deficits at the level of information processing are highly debated. For example, central to this debate is the question of whether the core deficit of ASD is in mentalizing (Baron-Cohen et al., 1985; Baron-Cohen, 1995) conceptualized as a modular-based mechanism, or whether it is in executive function more generally (Russell, 1997)-or some product of complex interactions between the former and latter in development, as detailed in recent studies on executive dysfunction in ASD (Hill, 2004; Hill and Bird, 2006). 
An important pattern in the task performance of ASD participants (within- and betweensubjects) is that they tend to perform tasks heterogeneously (Hill and Bird, 2006; Towgood et al., 2009); they perform with impaired as well as supra-normal performance: heterogeneous deficits are to be expected if brain regions develop atypically. Some put forward the possibility that there are functionally disorganized brain systems in the prefrontal cortex (Minshew et al., 1997): the anatomical proximity of rostral and caudal medial Brodmann Area 10 in development renders the idea of functional disorganization in this prefrontal region highly plausible. Recent studies have instantiated functional disorganization along the rostral-caudal axis of functional specialization in rostromedial prefrontal cortex (Gilbert et al., 2008, 2009). More specifically, these studies found that, in groups matched for age, gender, and IQ, rostromedial prefrontal cortex was functionally reorganized, in that recruitment of caudal rostromedial prefrontal cortex (i.e., base-region localization of mentalizing) was markedly greater compared to control groups during tasks that tested the rostral attentional gateway (i.e., attentional switching), particularly the functions unique to the anterior region of rostromedial prefrontal cortex, namely stimulus-oriented thought, and the anterior rostromedial prefrontal cortex was under-recruited in these studies. This finding is consistent with other findings of functional underconnectivity in ASD (Bird et al., 2006; Castelli et al., 2002; Just et al., 2007).

To diagnose a patient with ASD as defined in the classification, the clinician must use diagnostic criteria that associate persisting deficits in communication and social interactions with narrow and repetitive behaviors, interests or activities. These two features manifest in childhood, affect social, academic or professional functioning, and are not better explained by an intellect developmental disorder or a global developmental delay (American Psychiatric Association, 2013). Although initially considered as an abnormal condition, several works have showed how the distribution of autistic traits across the population is continuous (Wing, 1988; Constantino and Todd, 2003), and people diagnosed with autism score at the extreme end of the distribution when autistic traits are measured (Baron-Cohen, 2010).

The Autism-Spectrum Quotient (AQ) is a widely used self-administered questionnaire that provides a quantified evaluation of the degree to which an adult with a normal intelligence quotient presents with signs of ASD. This measurement tool assesses the respondent's behaviors, preferences and cognition based on five domains of autistic functioning: social skill, attention switching, attention to detail, communication, and imagination; autistic traits are considered when the respondent shows poor social skill, strong attention focus, exceptional attention to detail, poor communication and poor imagination (Baron-Cohen et al., 2001). The AQ was translated in several languages, such as Italian (Ruta et al., 2012), Dutch (Hoekstra et al., 2008), Chinese (Lau et al., 2013), Japanese (Wakabayashi et al., 2006), Turkish (Kose et al., 2013), Polish (Pisula et al., 2013), and Persian (Mohammadi et al., 2012), and French (Kempenaers et al., 2017), and its structural validity was studied using exploratory and confirmatory factor analysis (EFA and CFA) in the different translations. Although initial evidence supporting the structural validity of $A Q$, several studies reported doubts regarding the factorial aspects of the tool (Hoekstra et al., 2011; Hurst et al., 2007), with multiple works not obtaining the five-factor original model to fit in student data (Hoekstra et al., 2011; Kloosterman et al., 2011; Lau et al., 2013); most of which argued for shorter versions of the questionnaire. An abridged ten-item version of the AQ was therefore created including "red flag" items for autism screening in general practice (Allison et al., 2012). Despite criticism, the AQ is the most commonly used measurement tool for autistic traits 
detection and is widely cited in scientific research (Ruzich et al., 2015).

The AQ was conceived based on the assumption that autistic traits are measurable consequences of an underlying cause - that is, ASD. There is growing evidence pointing towards defects in neuronal migration and the consequent malformation and malfunction of various brain circuits as etiology of several brain disorders such as ASD (Pan et al., 2019), which then presents itself through several autistic traits; as understood in the latent variable model of mental illness, however, autistic traits do not actively participate to the clinical manifestation in subjects, as they are effects of the construct they stem from. Recent works, however, have shown how behaviors in the autism spectrum reinforce each other (i.e. present a mutual influence): learning strategies can therefore be adopted as to correct the social behavior of people with autistic traits, and brain responses to social stimuli can be altered, especially if an early intervention is conducted (Schuetze et al., 2017). From an ontological point of view, this changes the attribute of autistic traits, that evolve from measurable consequences of ASD to participate in its clinical presentation.

In the last decade, network analysis has affirmed itself as a new way of analyzing data in psychiatry and psychology, which allows the conception of of mental disorders or constructs as emerging from a complex system of mutually infleuencing components (Borsboom and Cramer, 2013). This novel theoretical and methodological approach has been widely used to explore a variety of constructs including depression (Mullarkey et al., 2018), obsessive compulsive disorder (McNally et al., 2017), empathy (Briganti et al., 2018), personality (Costantini et al., 2015), alexithymia (Briganti and Linkowski, 2019c), self-worth (Briganti et al., 2019), posttraumatic stress disorder (Fried et al., 2018; Phillips et al., 2018), resilience (Fritz et al., 2018; Briganti and Linkowski, 2019b), and narcissism (Di Pierro et al., 2019; Briganti and Linkowski, 2019a).

Researchers usually analyze mental disorders and constructs as network composed of items - answers of the observed group to a given questionnaire, such as the AQ. Conceiving mental disorders as networks is interesting in clinical practice, since relevant components in the proposed model could serve as a target for intervention (Fried et al., 2018). Most of the recent empirical works using network analysis compute the unobserved interactions between psychological components as regularized partial correlations (Epskamp and Fried, 2018): this is commonly achieved with $\ell_{1}$-regularization (Friedman et al., 2014), which is also known as the "least absolute shrinkage and selection operator" or "lasso," which pushes the smaller estimates in the network structure to zero and therefore renders a sparse (or conservative) network. The dominant lasso procedure used in network papers is the graphical lasso that associates the $\ell_{1}$-regularization with an Extended Bayesian Information Criterion to determine a tuning parameter for the Gaussian Graphical Model (i.e. the network) $\lambda$ (Chen and Chen, 2008). However, recent work (see for example Williams et al., 2019) reported how the regularization of network estimates can be inconsistent for model selection, and does not provide evidence for the null hypothesis (i.e. evidence for no effect); the latter consequence is of great importance because one of the core ideas of estimating network structure is to uncover the conditional independence structure of a construct, and therefore zero partial correlations among the variables composing the network.

Recently, Bayesian methodology has been introduced for the estimation of network structures. This new methodological framework allows for the estimation of Gaussian Graphical Models with posterior probabilities that can assess the conditional dependent and indepen- 
dent relations among components of a network with a decision rule that can be calibrated to a desired level of specificity (Williams, 2018). The Bayesian approach to the estimation of network structures shows advantages when determining conditional independence relations based on a network estimation where the threshold for selection depends on Bayes factors (Williams and Mulder, 2019): this technique allows for studying the amount of evidence for no effect in a given structure, which is useful to assess the uncertainty of estimates. Despite offering such advantages, Bayesian methodology for the estimation of Gaussian Graphical Models has never been used in empirical works concerning network analysis in psychological or psychiatric research.

Inspired by these new advances in network analysis that has been applied in many fields of psychiatry and psychology, we apply the network methodology to autistic traits. The aim of the present work is therefore to perform a network analysis of the autistic traits as presented in the 50-item version of the AQ with the Bayesian inference methods that have been recently introduced (Williams, 2018), in a sample of 649 university students. Autism has recently been analyzed through the lens of network analysis in relationship with depression (van Heijst et al., 2019); however, to our knowledge, this is the first time that the AQ in its full 50-item version is explored with the network methodology, therefore expanding this conceptual and methodological framework to the construct of autistic traits. In this work, we will first estimate the conditional dependence structure of the autistic traits network. Second, we will estimate node predictability, i.e. point-estimate and confidence intervals for variance explained in all items, to measure their overall connectedness to other items in the questionnaire. Third, we will explore sex differences among female and male subjects from this study. The data set for this study is available upon reasonable request addressed to the first author of this study. The protocol for this study was approved by the ethical committee of Hopital Erasme (Erasme Teaching Hospital) in Brussels, Belgium.

\section{Method}

\subsection{Data set}

Our data set is composed of 649 university students from the French-speaking region of Belgium. Subjects were 17 to 25 years old $(\mathrm{M}=19.3, \mathrm{SD}= \pm 1.49) ; 58 \%$ were female and $42 \%$ were male.

\subsection{Measurement}

The AQ is composed of 50 items that assess autistic traits based on five domains: social skill, attention switching, attention to detail, communication, and imagination. The minimum score for each item is 1 ("Definitely disagree") and the maximum score is 4 ("Definitely agree"); approximately half of the items in the questionnaire are to be reverse-scored (BaronCohen et al., 2001). 


\subsection{Network analysis}

\subsubsection{Software and packages}

We used the software $\mathrm{R}$ for statistical computing (version 3.6.1, open source, available at https://www.r-project.org/). The package used to carry out the analysis is BGGM (Williams and Mulder, 2019).

\subsubsection{Network estimation}

We estimated a Gaussian Graphical Model (GGM) with Bayesian methods (Williams, 2018), that is, a partial correlation network for the 50 items in the AQ. The GGM is calculated as the inverse-covariance matrix: it is a network that includes a set of nodes that correspond to the autistic traits in the AQ and a set of edges that connect the nodes in the network. If two nodes are connected, that means they are conditionally dependent given all other nodes in the network (i.e their partial correlation is nonzero). In the network of autistic traits, if two nodes A and B are connected, it means for instance that if the observed group scored high on trait $\mathrm{A}$, then the observed group is also more likely to score high on trait $\mathrm{B}$, and vice versa, controlling for other nodes in the network (Briganti et al., 2018). Each edge in the network has a weight representing the strength of association between two autistic traits; edges can be positive (and therefore represent a positive association) or negative (denoting a negative association). The estimation of network with Bayesian methods allows for providing evidence for the hypothesis that best predicts the observed data. For instance, when testing for conditional dependence relationships among nodes, providing a Bayes Factor (BF) between 3 and 20 as a cut-off value is associated with positive evidence, while a BF i 20 is associated with strong evidence (Kass and Raftery, 1995). For the network estimation in this paper, a BF of 20 was used.

\subsubsection{Node predictability and sex differences}

We computed Bayesian $R^{2}$ for the fifty autistic traits in the network, which represents the percentage of variance explained of a given autistic trait with all other autistic traits in the network (Williams and Mulder, 2019): this measure is commonly defined "node predictability" in the network literature (Haslbeck and Fried, 2017) as it can be interpreted as how well a node connects to other nodes in the network, or the self-determination of the network. Node predictability can be understood as the upper bound of controllability: if one assumes that all edges for a given node are directed toward that node, predictability provides an estimate of how much influence we can have on the given node via all other nodes (Briganti et al., 2019). Because the estimation of node predictability results in a distribution, we compared the predictability estimates of females and male subjects in our data set to detect sex differences in the network structures. 


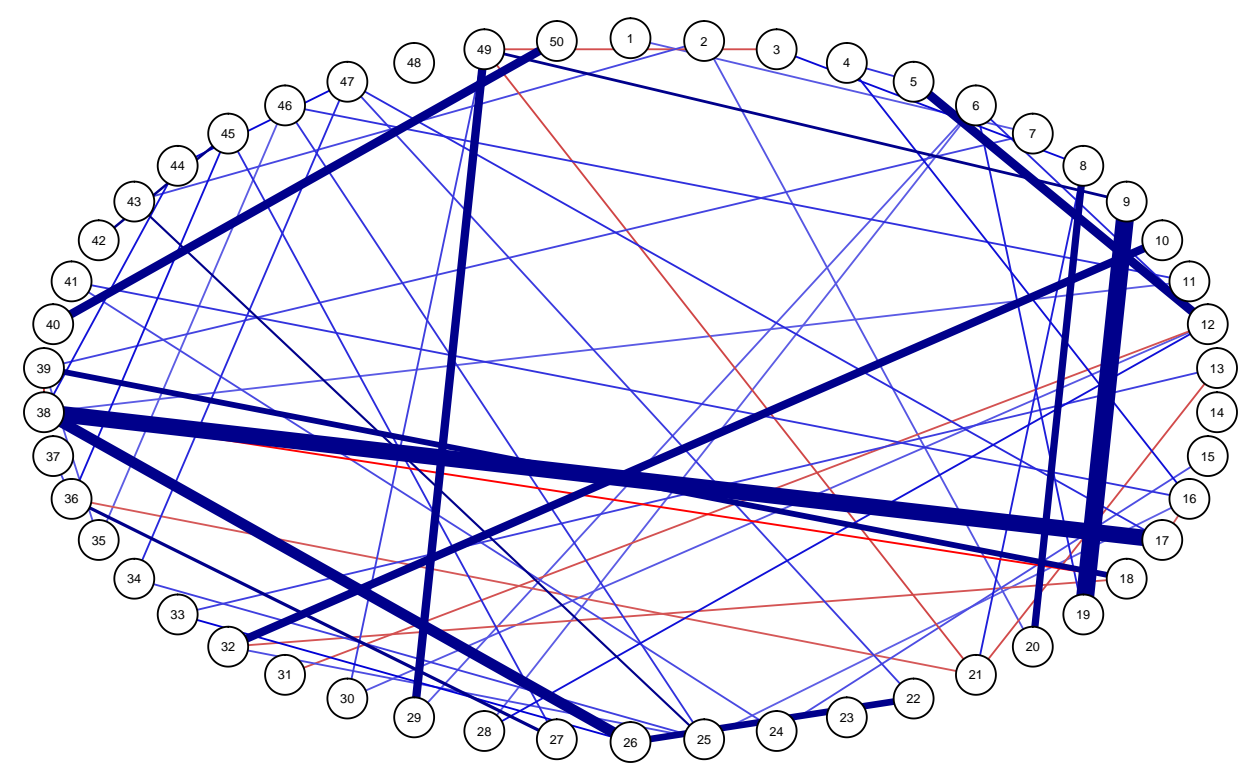

Figure 1: Autistic traits $(N=649)$ partial correlation network with the 50 items from the AQ. Each edge (connection between nodes) is denoted by a weight represented by thickness and color saturation. 


\section{Results}

\subsection{Partial correlation network}

The autistic traits partial correlation network is reported in figure 1 . The strongest connections are found between items that measure the same (or similar) autistic traits: for example item 38 ("I am good at social chit-chat", reversed) is strongly connected to item 17 ("I enjoy social chit-chat," reversed) and item 26 ("I frequently find that I don't know how to keep a conversation going"). Two other examples of this phenomenon can be found in the connections between item 8 ("When I'm reading a story, I can easily imagine what the characters might look like," reversed) and 20 ("When I'm reading a story, I find it difficult to work out the characters' intentions") as well as between item 9 ("I am fascinated by dates") and 19

("I am fascinated by numbers").

However, connections between items that measure different autistic traits can also be found in the network: for instance, 27 ("I find it easy to read between the lines when someone is talking to me," reversed) and 36 ("I find it easy to work out what someone is thinking or feeling just by looking at their face," reversed), 11 ("I find social situations easy," reversed) and 38 ("I am good at social chit-chat," reversed), 35 ("I am often the last to understand the point of a joke") and 46 ("New situations make me anxious").

Several negative connections can also be found in the network, such as the ones between items 18 ("When I talk, it isn't always easy for others to get a word in edgeways") and 32 ("I find it easy to do more than one thing at once," reversed), 21 ("I don't particularly enjoy reading fiction," reversed) and 49 ("I am not very good at remembering people's date of birth," reversed), 12 ("I tend to notice details that others do not," reversed) and 31 ("I know how to tell if someone listening to me is getting bored").

\subsection{Node predictability}

The computed values for node predictability in the network are reported in figure 2 . Item 38 ("I am good at social chit-chat", reversed) has the highest $R^{2}$ score $(54 \%)$ in the network, while item 41 ("I like to collect information about categories of things") has the lowest $R^{2}$ score $(9 \%)$ in the network. The average $R^{2}$ score is $20 \%$.

\subsection{Sex differences}

Figure 3 represents sex differences in node predictability. The autistic trait network structures are statistically different in female and male subjects, with a Kullback-Leibler divergence value (that translates the difference between the two node predictability distributions) of 5.8, and a $p$-value of 0 .

A heat map is reproduced in figure 4 that represents which specific edges are different in the female and male network structure (and the respective amount of evidence supporting the difference, represented by a variation in $\mathrm{BF}$ ). For instance, there is a moderate amount of evidence supporting sex differences for the specific edges between items 14 ("I find making up stories easy") and 22 ("I find it hard to make new friends"), with a Bayes Factor of 115, item 17 ("I enjoy social chit-chat") and 34 ("I enjoy doing things spontaneously"), with a Bayes Factor of 90. 


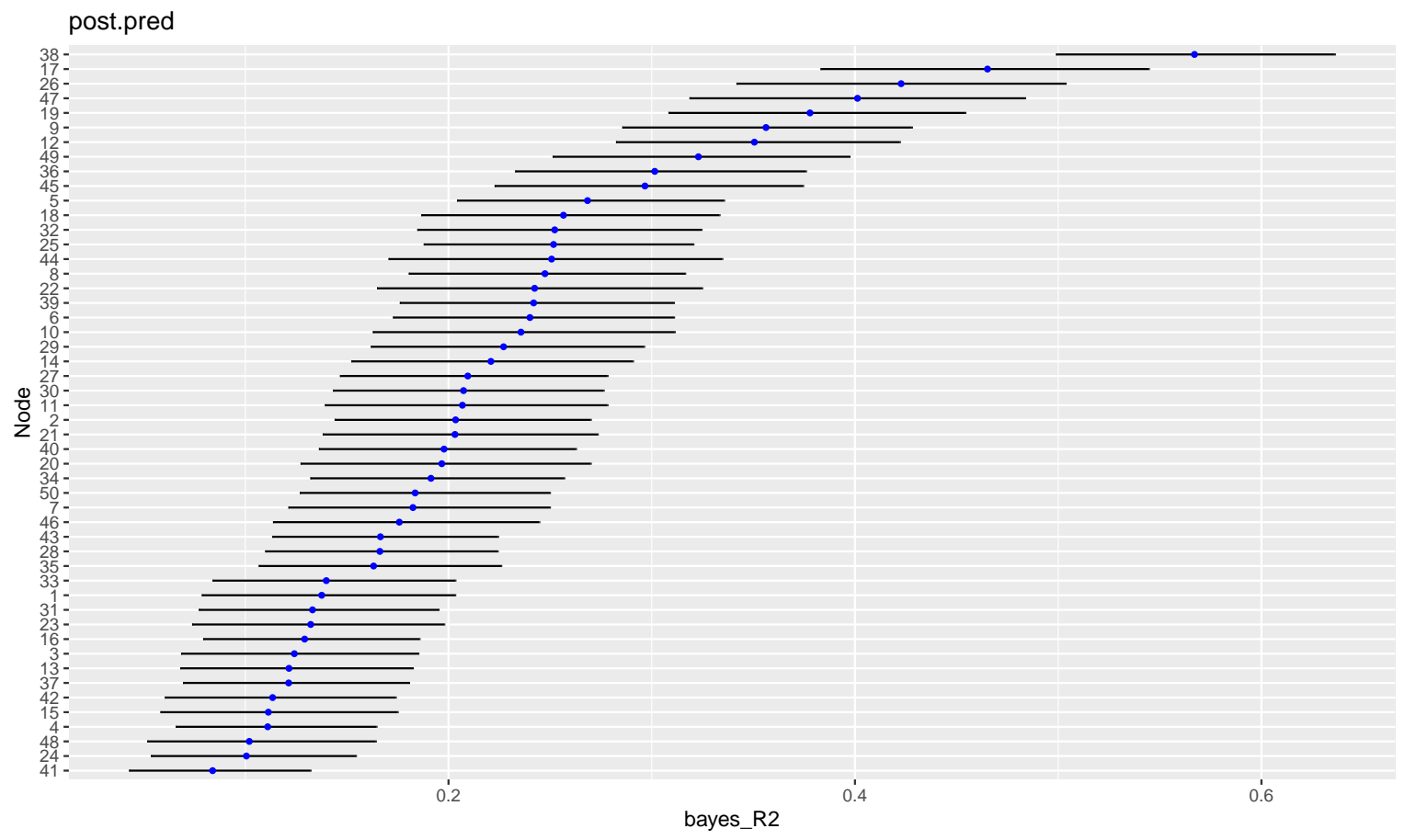

Figure 2: Node predictability (Bayesian $R^{2}$ ) for the 50 nodes in the partial correlation network.

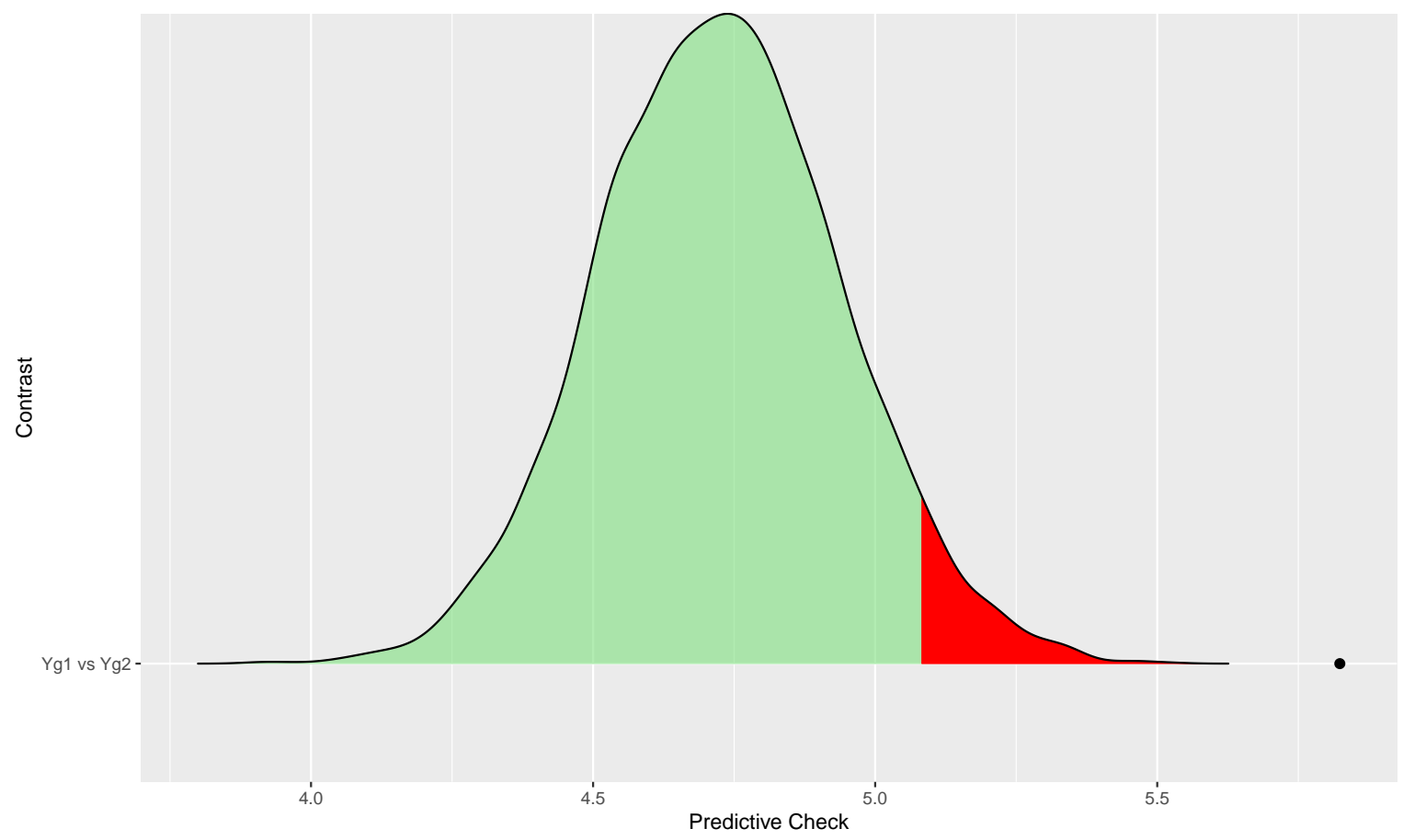

Figure 3: Sex differences in the network of autistic traits. 


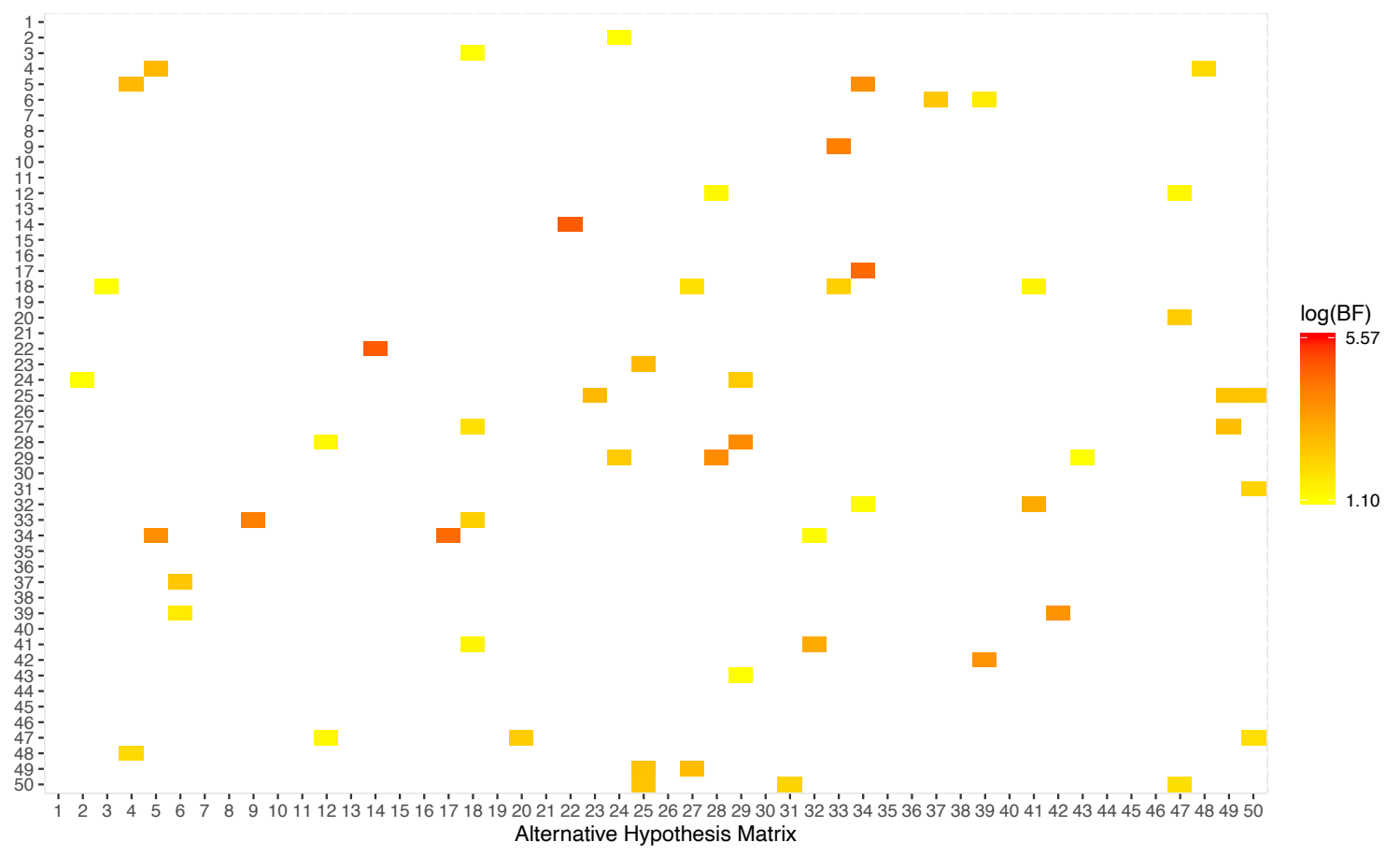

Figure 4: Heat map reporting the edges that are statistically different in regard to the sex of participants. The darker the color, the higher the evidence.

The number of edges that are statistically different in regard to the sex of participants variates among the items in the $\mathrm{AQ}$ and are reported in figure 5. Item 17 ("I enjoy social chit-chat") presents the highest number of edges in that regard.

\section{Discussion}

This is the first work tackling the exploration of autistic traits through the lenses of Bayesian network analysis and using the full 50-item version of the largely validated AQ (Baron-Cohen et al., 2001). New methods introduced in recent Bayesian network literature (Williams, 2018) allow for the estimation of conditional relationships among nodes with Bayesian methods, including adopting a cut-off value to provide consistent evidence of the existence of such relationships. Studying how items from the AQ connect sheds new information on the important construct such as the one representing autistic traits. Even in a student sample, investigating autistic traits through the lenses of network analysis is relevant because "non-autistic" individuals may still present autistic traits, which are continuously distributed across the general population.

The strongest connections in the network are found between items that measure similar autistic traits: this is a recurrent phenomenon in network analysis of psychometric scales (Briganti et al., 2018, 2019; Briganti and Linkowski, 2019b,a), which substantially influences the network predictability.

Additional insight to the construct is mostly brought by connections between items that 


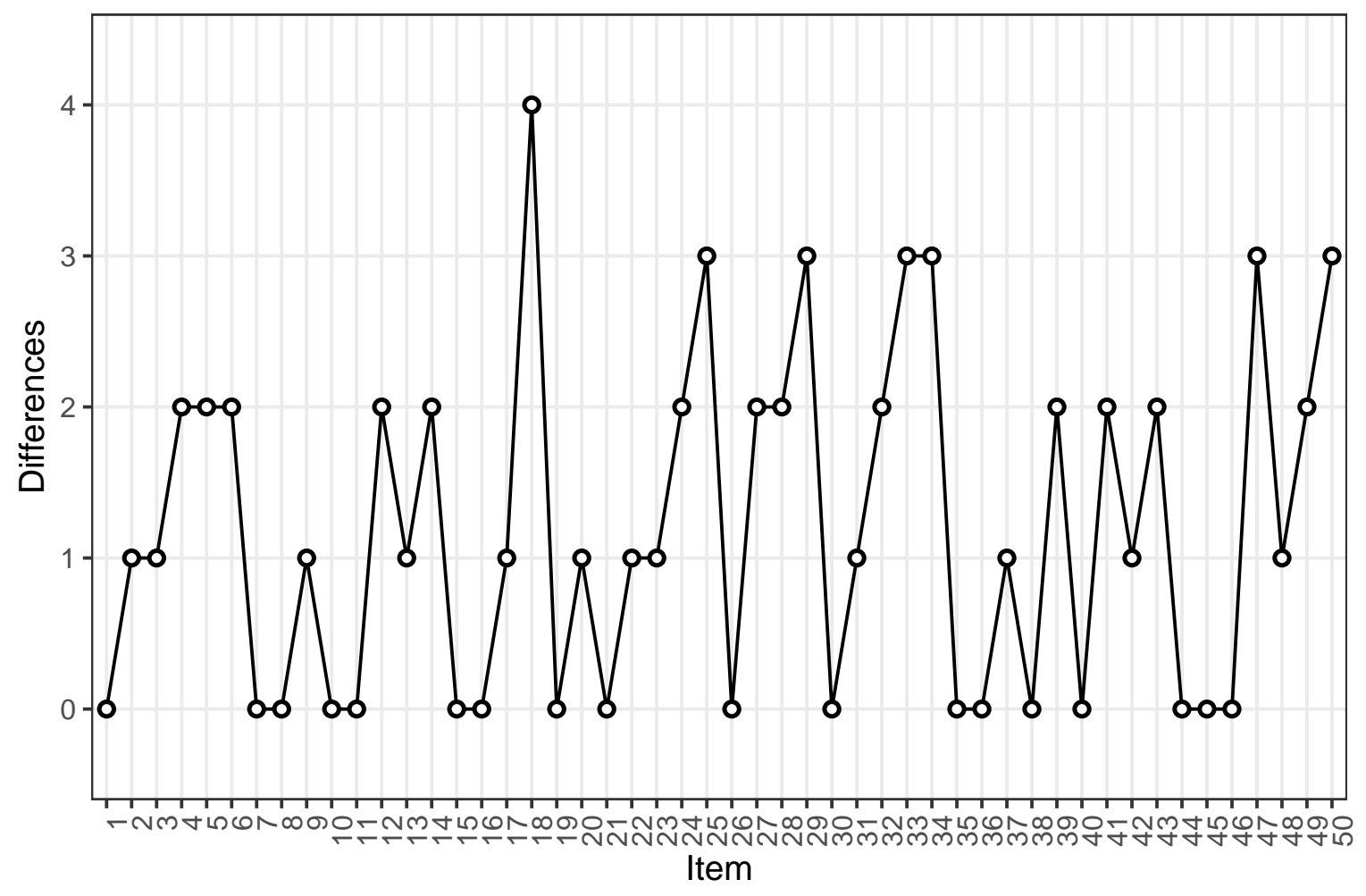

Figure 5: Plot reporting for each node the number of edges that are statistically different in regard to the sex of participants. 
measure different autistic traits, which are also found in the network, but their connection strength is proportionally lower. From a network perspective, a connection between different autistic traits such as the one between item 35 ("I am often the last to understand the point of a joke") and 46 ("New situations make me anxious") is interesting if interpreted on a clinical level. For instance, if a patient with autistic traits is bothered that he often lacks the ability to understand jokes, it might be interesting for the clinician to investigate if and how new situations make that same patient anxious, while taking into account other autistic traits.

The presence of several negative connections in the network of autistic traits is an interesting finding: the model is not overall positively connected, which is unusual from a latent variable model perspective in which all items are considered as interchangeable measures of the latent variable (in this case, the autism spectrum). This means several autistic traits are inversely correlated. Negative connections occur between items from different domains (i.e. different facets of the autism spectrum). For instance and in our sample, the average individual presenting the autistic trait of being good at remembering people's date of birth (item 49) is less likely to present the autistic trait of enjoying reading fiction (item 21).

The highest $R^{2}$ score in the network belongs to item 38, which measures an autistic trait related to social skills. From a network point of view this can be interpreted as the social skills trait being the domain that best predicts (or is predicted by) the rest of autistic traits, since it is the most interconnected trait. On the other hand, item 41, an autistic trait related to the domain of imagination, has the lowest $R^{2}$ score in the network, which means it is poorly connected to other autistic traits. The average $R^{2}$ score is $20 \%$, which means that on average, nodes in the network of autistic traits share $20 \%$ of variance with other nodes.

Sex differences are found between the network of female and male subjects. This finding supports extensive research done in the past few years which points to high AQ scores being preponderant in male subjects (Ferri et al., 2018; Baron-Cohen et al., 2014); this study however reports a difference in network connectivity, which is an additional information never explored before.

Our findings must be interpreted in light of certain limitations: two of them are hereby described. First, the data set used in this study is composed of Belgian university students, which likely limits the replicability of our findings in other sample, including samples with people diagnosed with autism. Second, the uncertainty regarding the true network structure of autistic traits must be taken into account: whereas it is possible to detect edges (non-zero effects) with large uncertainty, inferring null effects (zero effects or the lack of connection between two nodes) requires a much greater sample, especially in our case, where 50 items are analyzed.

Further studies may endeavor to replicate our findings in other samples, including people diagnosed with autism.

\section{References}

Allison, C., Auyeung, B., and Baron-Cohen, S. (2012). Toward brief "red flags" for autism screening: the short autism spectrum quotient and the short quantitative checklist in 1,000 cases and 3,000 controls. Journal of the American Academy of Child \& Adolescent Psychiatry, 51(2):202-212. 
American Psychiatric Association (2013). Diagnostic and Statistical Manual of Mental Disorders. American Psychiatric Association, fifth edition.

Baron-Cohen, S. (1995). Mindblindness: An Essay on Autism and Theory of Mind (Learning, Development, and Conceptual Change). MIT Press.

Baron-Cohen, S. (2010). Progress in brain research: Sex differences in the human brain, their underpinnings and implications. Progress in Brain Research, 186:167-175.

Baron-Cohen, S., Cassidy, S., Auyeung, B., Allison, C., Achoukhi, M., Robertson, S., Pohl, A., and Lai, M.-C. (2014). Attenuation of Typical Sex Differences in 800 Adults with Autism vs. 3,900 Controls. PLoS ONE, 9(7).

Baron-Cohen, S., Leslie, A. M., and Frith, U. (1985). Does the autistic child have a "theory of mind"? Cognition, 21(1):37-46.

Baron-Cohen, S., Wheelwright, S., Skinner, R., Martin, J., and Clubley, E. (2001). The autism-spectrum quotient (AQ): evidence from Asperger syndrome/high-functioning autism, males and females, scientists and mathematicians. Journal of Autism and Developmental Disorders, 31(1):5-17.

Bird, G., Catmur, C., Silani, G., Frith, C., and Frith, U. (2006). Attention does not modulate neural responses to social stimuli in autism spectrum disorders. Neuroimage, 31(4):16141624 .

Borsboom, D. and Cramer, A. O. (2013). Network Analysis: An Integrative Approach to the Structure of Psychopathology. Annual Review of Clinical Psychology, 9(1):91-121.

Briganti, G., Fried, E. I., and Linkowski, P. (2019). Network analysis of Contingencies of Self-Worth Scale in 680 university students. Psychiatry Research, 272:252-257.

Briganti, G., Kempenaers, C., Braun, S., Fried, E. I., and Linkowski, P. (2018). Network analysis of empathy items from the interpersonal reactivity index in 1973 young adults. Psychiatry Research, 265:87-92.

Briganti, G. and Linkowski, P. (2019a). Exploring network structure and central items of the narcissistic personality inventory. International Journal of Methods in Psychiatric Research, n/a:e1810.

Briganti, G. and Linkowski, P. (2019b). Item and domain network structures of the Resilience Scale for Adults in 675 university students. Epidemiology and Psychiatric Sciences, pages $1-9$.

Briganti, G. and Linkowski, P. (2019c). Network approach to items and domains from the toronto alexithymia scale. Psychological Reports, page 0033294119889586.

Castelli, F., Frith, C., Happé, F., and Frith, U. (2002). Autism, asperger syndrome and brain mechanisms for the attribution of mental states to animated shapes. Brain, 125(8):18391849. 
Chen, J. and Chen, Z. (2008). Extended Bayesian information criteria for model selection with large model spaces. Biometrika, 95(3):759-771.

Constantino, J. N. and Todd, R. D. (2003). Autistic traits in the general population: a twin study. Archives of general psychiatry, 60(5):524-530.

Costantini, G., Richetin, J., Borsboom, D., Fried, E. I., Rhemtulla, M., and Perugini, M. (2015). Development of Indirect Measures of Conscientiousness: Combining a Facets Approach and Network Analysis. European Journal of Personality, 29(5):548-567.

Di Pierro, R., Costantini, G., Benzi, I. M. A., Madeddu, F., and Preti, E. (2019). Grandiose and entitled, but still fragile: A network analysis of pathological narcissistic traits. Personality and Individual Differences, 140:15-20.

Epskamp, S. and Fried, E. I. (2018). A tutorial on regularized partial correlation networks. Psychological Methods, 23(4):617-634.

Ferri, S. L., Abel, T., and Brodkin, E. S. (2018). Sex Differences in Autism Spectrum Disorder: A Review. Current psychiatry reports, 20(2):9.

Fried, E. I., Eidhof, M. B., Palic, S., Costantini, G., Huisman-van Dijk, H. M., Bockting, C. L. H., Engelhard, I., Armour, C., Nielsen, A. B. S., and Karstoft, K.-I. (2018). Replicability and Generalizability of Posttraumatic Stress Disorder (PTSD) Networks: A Cross-Cultural Multisite Study of PTSD Symptoms in Four Trauma Patient Samples. Clinical Psychological Science.

Friedman, J., Hastie, T., and Tibshirani, R. (2014). glasso: Graphical lasso- estimation of Gaussian graphical models.

Fritz, J., Fried, E. I., Goodyer, I. M., Wilkinson, P. O., and van Harmelen, A.-L. (2018). A Network Model of Resilience Factors for Adolescents with and without Exposure to Childhood Adversity. Scientific Reports, 8(1):15774.

Gilbert, S. J., Bird, G., Brindley, R., Frith, C. D., and Burgess, P. W. (2008). Atypical recruitment of medial prefrontal cortex in autism spectrum disorders: An fmri study of two executive function tasks. Neuropsychologia, 46(9):2281-2291.

Gilbert, S. J., Meuwese, J. D., Towgood, K. J., Frith, C. D., and Burgess, P. W. (2009). Abnormal functional specialization within medial prefrontal cortex in high-functioning autism: a multi-voxel similarity analysis. Brain, 132(4):869-878.

Haslbeck, J. M. B. and Fried, E. I. (2017). How predictable are symptoms in psychopathological networks? A reanalysis of 18 published datasets. Psychological Medicine, 47(16):27672776 .

Hill, E. L. (2004). Evaluating the theory of executive dysfunction in autism. Developmental review, 24(2):189-233.

Hill, E. L. and Bird, C. M. (2006). Executive processes in asperger syndrome: Patterns of performance in a multiple case series. Neuropsychologia, 44(14):2822-2835. 
Hoekstra, R. A., Bartels, M., Cath, D. C., and Boomsma, D. I. (2008). Factor structure, reliability and criterion validity of the Autism-Spectrum Quotient (AQ): a study in Dutch population and patient groups. Journal of Autism and Developmental Disorders, $38(8): 1555-1566$.

Hoekstra, R. A., Vinkhuyzen, A. A. E., Wheelwright, S., Bartels, M., Boomsma, D. I., BaronCohen, S., Posthuma, D., and van der Sluis, S. (2011). The construction and validation of an abridged version of the autism-spectrum quotient (AQ-Short). Journal of Autism and Developmental Disorders, 41(5):589-596.

Hurst, R. M., Mitchell, J. T., Kimbrel, N. A., Kwapil, T. K., and Nelson-Gray, R. O. (2007). Examination of the reliability and factor structure of the Autism Spectrum Quotient (AQ) in a non-clinical sample. Personality and Individual Differences, 43(7):1938-1949.

Just, M. A., Cherkassky, V. L., Keller, T. A., Kana, R. K., and Minshew, N. J. (2007). Functional and anatomical cortical underconnectivity in autism: evidence from an fmri study of an executive function task and corpus callosum morphometry. Cerebral cortex, 17(4):951-961.

Kass, R. E. and Raftery, A. E. (1995). Bayes Factors. Journal of the American Statistical Association, 90(430):773-795.

Kempenaers, C., Braun, S., Delvaux, N., and Linkowski, P. (2017). The assessment of autistic traits with the Autism Spectrum Quotient: Contribution of the French version to its construct validity. European Review of Applied Psychology, 67(6):299-306.

Kloosterman, P. H., Keefer, K. V., Kelley, E. A., Summerfeldt, L. J., and Parker, J. D. A. (2011). Evaluation of the factor structure of the Autism-Spectrum Quotient. Personality and Individual Differences, 50(2):310-314.

Kose, S., Bora, E., Erermiş, S., Özbaran, B., Bildik, T., and Aydın, C. (2013). Broader autistic phenotype in parents of children with autism: Autism Spectrum Quotient-Turkish version. Psychiatry and Clinical Neurosciences, 67(1):20-27.

Lau, W. Y.-P., Gau, S. S.-F., Chiu, Y.-N., Wu, Y.-Y., Chou, W.-J., Liu, S.-K., and Chou, M.-C. (2013). Psychometric properties of the Chinese version of the Autism Spectrum Quotient (AQ). Research in Developmental Disabilities, 34(1):294-305.

McNally, R. J., Mair, P., Mugno, B. L., and Riemann, B. C. (2017). Co-morbid obsessive-compulsive disorder and depression: a Bayesian network approach. Psychological Medicine, 47(7):1204-1214.

Minshew, N. J., Goldstein, G., and Siegel, D. J. (1997). Neuropsychologic functioning in autism: Profile of a complex information processing disorder. Journal of the International Neuropsychological society, 3(4):303-316.

Mohammadi, M. R., Zarafshan, H., and Ghasempour, S. (2012). Broader Autism Phenotype in Iranian Parents of Children with Autism Spectrum Disorders vs. Normal Children. Iranian Journal of Psychiatry, 7(4):157-163. 
Mullarkey, M. C., Marchetti, I., and Beevers, C. G. (2018). Using Network Analysis to Identify Central Symptoms of Adolescent Depression. Journal of Clinical Child $\mathscr{G}$ Adolescent Psychology, 0(0):1-13.

Pan, Y.-H., Wu, N., and Yuan, X.-B. (2019). Toward a better understanding of neuronal migration deficits in autism spectrum disorders. Frontiers in Cell and Developmental Biology, 7:205.

Phillips, R. D., Wilson, S. M., Sun, D., VA Mid-Atlantic MIRECC Workgroup, and Morey, R. (2018). Posttraumatic Stress Disorder Symptom Network Analysis in U.S. Military Veterans: Examining the Impact of Combat Exposure. Frontiers in Psychiatry, 9:608.

Pisula, E., Kawa, R., Szostakiewicz, L., Lucka, I., Kawa, M., and Rynkiewicz, A. (2013). Autistic traits in male and female students and individuals with high functioning autism spectrum disorders measured by the Polish version of the Autism-Spectrum Quotient. PloS One, 8(9):e75236.

Russell, J. E. (1997). Autism as an executive disorder. Oxford University Press.

Ruta, L., Mazzone, D., Mazzone, L., Wheelwright, S., and Baron-Cohen, S. (2012). The Autism-Spectrum Quotient-Italian version: a cross-cultural confirmation of the broader autism phenotype. Journal of Autism and Developmental Disorders, 42(4):625-633.

Ruzich, E., Allison, C., Smith, P., Watson, P., Auyeung, B., Ring, H., and Baron-Cohen, S. (2015). Measuring autistic traits in the general population: a systematic review of the Autism-Spectrum Quotient (AQ) in a nonclinical population sample of 6,900 typical adult males and females. Molecular Autism, 6:2.

Schuetze, M., Rohr, C. S., Dewey, D., McCrimmon, A., and Bray, S. (2017). Reinforcement learning in autism spectrum disorder. Frontiers in Psychology, 8:2035.

Towgood, K. J., Meuwese, J. D., Gilbert, S. J., Turner, M. S., and Burgess, P. W. (2009). Advantages of the multiple case series approach to the study of cognitive deficits in autism spectrum disorder. Neuropsychologia, 47(13):2981-2988.

van Heijst, B. F., Deserno, M. K., Rhebergen, D., and Geurts, H. M. (2019). Autism and depression are connected: A report of two complimentary network studies. Autism, page 1362361319872373 .

Volkmar, F. R., State, M., and Klin, A. (2009). Autism and autism spectrum disorders: diagnostic issues for the coming decade. Journal of Child Psychology and Psychiatry, and Allied Disciplines, 50(1-2):108-115.

Wakabayashi, A., Baron-Cohen, S., Wheelwright, S., and Tojo, Y. (2006). The AutismSpectrum Quotient (AQ) in Japan: A cross-cultural comparison. Journal of Autism and Developmental Disorders, 36(2):263-270.

Williams, D. R. (2018). Bayesian estimation for gaussian graphical models: Structure learning, predictability, and network comparisons. 
Williams, D. R. and Mulder, J. (2019). BGGM: A R Package for Bayesian Gaussian Graphical Models.

Williams, D. R., Rhemtulla, M., Wysocki, A. C., and Rast, P. (2019). On Nonregularized Estimation of Psychological Networks. Multivariate behavioral research, 54(5):719-750.

Wing, L. (1988). Aspects of autism: Biological research. Royal College of Psychiatrists. 